\title{
PERTINENCIA DE LOS PROGRAMAS PARA EL APRENDIZAJE DEL INGLÉS EN ADMINISTRACIÓN DE EMPRESAS, RECINTO NUEVA GUINEA
}

Ángel M. Dávila ${ }^{[1]}$

\section{Resumen}

Se han estudiado los programas para el aprendizaje del inglés en el 2009, con estudiantes del sexto cuatrimestre, modalidad regular, Administración de Empresas, Recinto URACCAN Nueva Guinea. Se analizó la pertinencia de enseñanza en 6 programas de inglés utilizados en esta licenciatura. Se caracterizaron las condiciones de infraestructura, materiales y medios didácticos empleados. También se describe qué motiva a los estudiantes a aprender y usar el idioma Inglés.

Los resultados indicaron que aunque se dispone de condiciones aceptables de infraestructura, profesores calificados y un currículo apropiado, se debe emprender esfuerzos para mejorar en cada uno de estos ámbitos con el fin de garantizar un nivel de comunicación significativa en el idioma inglés.

Palabras clave: aprendizaje significativo, motivación, materiales didácticos.

\section{Summary}

We have studied the programs for the learning of English in 2009, with students of the sixth cuatrimester, regular modality from Business Administration, Nueva Guinea Campus. We analyzed the pertinence of teaching English in 6 programs used in this degree. We characterized the conditions of the infrastructure, materials and teaching aids employed. The study also describes what motivates students to learn and use the English language.

The results indicated that although there are acceptable conditions of the infrastructure, qualified teachers and an appropriate curriculum, efforts should be undertaken to improve in each of these areas in order to ensure a meaningful level of communication in English.

Keywords: Meaningful learning, motivation, teaching materials.

${ }^{[1]}$ MSc. Docente URACCAN. Nueva Guinea, RAAS, Nicaragua. E-mail: angelmdavila@gmail.com 


\section{Introducción}

Este artículo es producto de los resultados finales de la tesis de maestría en Docencia Universitaria titulada: Pertinencia de los programas y condiciones brindadas para el aprendizaje del inglés. En nuestra actualidad resulta imprescindible aprender inglés. Cada día se emplea más en casi todas las áreas del conocimiento y desarrollo humano. Esta realidad ha hecho que las instituciones de Educación Superior a nivel mundial incorporen componentes curriculares enfocados a este lenguaje.

Nicaragua no es la excepción a esta realidad, se observa que día a día se crean academias e institutos de idiomas, cuyo objetivo es la enseñanza del inglés. Por esta razón, la Universidad de las Regiones Autónomas de la Costa Caribe Nicaragüense (URACCAN), en su Modelo Pedagógico, considera necesario: "aprender a comunicarse de forma adecuada en diferentes situaciones y con diferentes interlocutores en al menos dos lenguas" (URACCAN, 2004 p. 24).

Por consiguiente, se han incluido en el plan de estudios de la licenciatura en Administración de Empresas 6 programas de inglés, con el fin de "desarrollar habilidades en el inglés estándar, lenguaje utilizado en el campo de los negocios internacionales y la literatura más accesible." (URACCAN, 2003b, p. 5). El propósito de este estudio giró en torno a tres elementos que influyen en el proceso de enseñanza-aprendizaje del idioma inglés, específicamente con el estudiantado del sexto cuatrimestre, turno vespertino, modalidad regular en la URACCAN, Nueva Guinea, 2009: se investigaron aquellos aspectos que tienen que ver con la efectividad de los 6 programas de inglés, las condiciones de infraestructura, materiales y medios didácticos, asimismo sobre las motivaciones que incentivan al estudiantado a aprender el idioma inglés en la licenciatura en Administración de Empresas.

\section{Revisión de literatura}

\section{a. Teoría del aprendizaje significativo}

La teoría del aprendizaje significativo, es una teoría psicológica porque se ocupa de los procesos mismos que el individuo pone en juego para aprender. [...] pone el énfasis en lo que ocurre en el aula de clases cuando los estudiantes aprenden; en la naturaleza de ese aprendizaje; en las condiciones que se requieren para que éste se produzca; en sus resultados y, consecuentemente, en su evaluación (Ausubel, 1976) citado por Palmero 2004.

Según Magna (2009, p. 28), resalta que el éxito en la adquisición de lenguas extranjeras depende de factores biológicos y psicológicos, factores cognitivos y factores afectivos. Indica que hay que reflexionar sobre los factores sociales afectivos en el proceso de aprendizaje de una lengua extranjera. 


\section{b. Perspectiva interaccionista}

Williams y Burden (1999) citados por Martínez (2003), sostienen que: "El interaccionismo social destaca la naturaleza dinámica de la interacción entre profesores, estudiantes y tareas, y supone que el aprendizaje surge de las interacciones de unos con otros." Asimism, o sostiene que por regla general: "El aprendizaje de una lengua extranjera surge fundamentalmente mediante el desarrollo de la interacción comunicativa".

\section{Enfoque comunicativo}

Batista, Salazar y Febres (2001) expresan que:

aprender una lengua es aprender a comunicarse eficientemente. El objeto de enseñar una segunda lengua consiste en desarrollar equilibradamente la competencia gramatical, sociolingüística, discursiva y estratégica de la comunicación. Por esta razón se promueve el desarrollo de las cuatro destrezas de la lengua (escuchar-hablar y leerescribir) haciendo énfasis en el carácter funcional más que en aspectos gramaticales y situacionales (p. 18).

\section{c. Aportes sobre teoría curricular}

\section{Modelo Pedagógico de la URACCAN}

La URACCAN, asume un modelo pedagógico integral el cual debe recoger el pensamiento colectivo frente a nuestro quehacer: docencia, investigación y extensión comunitaria (URACCAN, 2004, p. 17).

\section{Estándares de valoración según el Consejo Nacional de Universidades sobre los programa de asignatura}

Introducción-Fundamentación: Breve descripción de la importancia de la asignatura en el proceso de formación del futuro profesional, sustenta y orienta los contenidos del programa hacia el perfil de formación. Estándares de valoración: Caracteriza la asignatura según tipo de formación. (General, básica y profesionalizante), ubica la asignatura dentro del cuerpo disciplinar al que corresponde, argumenta la ubicación de la asignatura dentro de la malla curricular (precedencia y prerrequisitos) y explicita el aporte de la disciplina y asignatura al logro de los conocimientos, habilidades y actitudes profesionales.

Objetivos generales: Son declaraciones de los propósitos metas que se desean alcanzar a través del desarrollo de los programas de asignaturas. Estándares de valoración: Están en correspondencia con los objetivos de la carrera y con el perfil 
profesional, incorporan aspectos cognoscitivos, procedimentales y actitudinales, las metas a través del desarrollo de la asignatura.

Plan temático: Es una tabla donde se organiza la asignatura por unidades y tiempo en horas, según régimen académico (semestral, cuatrimestral, trimestral, etc.). Criterios y estándares de valoración: El plan temático se corresponde con los objetivos del programa y establece un balance entre la teoría y la práctica.

Objetivos por unidad, competencias específicas: Concretan los aprendizajes específicos que propiciará el desarrollo de las capacidades propuestas en los objetivos y competencias generales de la asignatura. Estándares de valoración: Se corresponde con los objetivos y competencias generales de la asignatura, con los contenidos de las unidades, redactados en función de los estudiantes y son suficientes para identificar la unidad.

Estrategias metodológicas: Son sugerencias dirigidas a los docentes sobre los principios pedagógicos y metodológicos, que contribuyen a lograr los objetivos propuestos en una determinada asignatura. Estándares de valoración: Contienen los enfoques y métodos de enseñanza acordes a las características de las asignaturas, se corresponden con el modelo educativo institucional, incorpora el uso de los recursos didácticos en el abordaje de la asignatura para orientar a los docentes en las diversas asignaturas y modalidades.

Estrategias de evaluación de los aprendizajes: Es el conjunto de procedimientos que permiten medir el progreso de los estudiantes durante un período de tiempo. Estándares de valoración: Son coherentes con el proyecto educativo y el enfoque curricular, están en correspondencia con la naturaleza y particularidades de la asignatura, son variadas y se definen para los tres momentos de la evaluación: diagnóstica, formativa y sumativa.

Bibliografía: Son los textos consultados por el docente para la elaboración del programa y la recomendación de textos básicos y de consultas para el desarrollo del programa. Pueden ser también revistas y artículos impresos o electrónicos. Deben estar actualizados, menos de 10 años de haber sido publicados.

\section{Materiales y Métodos}

El estudio se desarrolló en el recinto URACCAN Nueva Guinea. Los sujetos informantes fueron el $100 \%$ de los estudiantes de Administración de Empresas, sexto cuatrimestre, turno vespertino, modalidad regular, 3 docentes que impartían los programas de inglés, y 2 autoridades de la URACCAN. Las fuentes secundarias tales como bibliográficas y webgrafía, sustentan la cientificidad del estudio. 
Esta investigación es cualitativa y tuvo un carácter descriptivo. La dimensión fue de corte transversal, porque se ubicó sólo en un momento del problema correspondiente al 2009. Se describió la pertinencia de los programas de asignatura, motivaciones y las condiciones de infraestructura, medios y materiales didácticos que se brindaron a los discentes para el aprendizaje significativo del inglés como segunda lengua.

Las técnicas de información fueron las entrevistas estructuradas, revisión de contenido y la encuesta escala Likert. El análisis de la información de las entrevistas se llevó a cabo a través de categorías, según los objetivos propuestos. Para los datos estadísticos se aplicó el programa informático Microsoft Word y los datos se procesaron con el programa estadístico SPSS.

\section{Resultados y discusión}

\section{Coherencia entre los textos y la enseñanza-aprendizaje del inglés}

El Modelo Pedagógico de URACCAN expresa que uno de los componentes fundamentales de esta Alma Máter es el currículo donde existe coherencia entre los programas de inglés, los textos, enfoque metodológico y el perfil de egreso. Entre otras, se pueden mencionar las siguientes características sobre el currículo: "relación permanente entre la teoría y la práctica, vistas como una unidad; la reflexión constante sobre el desempeño profesional futuro," "basado en valores científicos, tecnológicos, culturales, históricos, étnicos, lingüísticos," "actualizado de acuerdo con el avance de la ciencia y la tecnología," y además, ser "pertinente, integrante e integral." (URACCAN, 2004).

\section{Introducción de los programas de inglés}

Según el CNU (2008), cada programa de asignatura debe explicitar en la introducción el aporte de la disciplina y asignatura al logro de los conocimientos, habilidades y actitudes de profesionalización. Además, de caracterizar la asignatura según tipo de formación y ubicación dentro de la malla curricular. Sin embargo, los resultados muestran que ninguno de los 6 programas de inglés posee una introducción donde se mencionen estos aspectos.

Esto significa que los docentes que desarrollan estos programas, se guían sólo por los objetivos generales y objetivos por unidad de cada programa. Por lo que se infiere que si los programas de inglés no cuentan con esta información, el personal docente no tendrá un panorama claro del impacto de cada programa en el proceso educativo del idioma inglés.

En relación a la secuencia y coherencia lógica que debe seguir el proceso enseñanzaaprendizaje, Palmero (2004), en la teoría sobre el aprendizaje significativo define este tipo de aprendizaje como: 
Los nuevos conocimientos que se incorporan en forma sustantiva en la estructura cognitiva del estudiante. Esto se logra cuando el estudiante relaciona los conocimientos nuevos con los conocimientos que ya poseía; sin embargo es necesario que el estudiante se interese por lo que se le está enseñando.

\section{Objetivos y enfoque metodológico de los programas de inglés}

Por otro lado, los objetivos generales de los 6 programas de inglés que fueron analizados no se corresponden con los objetivos específicos y el perfil de egreso del currículo.. Además, cada programa se diseñó como único sin tomar en cuenta el anterior. Por ejemplo, el programa de inglés IV está elaborado en base a tres textos básicos: Intercom Book No. 4, Business Basic y Super Goal Book No. 2; sin embargo; las unidades tomadas de cada libro son independientes, o sea, no hay secuencia del aprendizaje anterior con el siguiente.

El único espacio donde se hace referencia al aprendizaje del inglés es en uno de los párrafos de la descripción del currículo, donde se expresa que: "Los estudiantes deben desarrollar habilidades en el inglés estándar ya que es la lengua utilizada en el campo de los negocios internacionales y la literatura más accesible de dicha titulación se publica en esta lengua (URACCAN, 2003).

A este respecto el CNU (2008), argumenta que los objetivos generales "expresan los conocimientos como conjunto de saberes que integran la asignatura, así mismo, el desarrollo de habilidades y actitudes que los estudiantes deben lograr en correspondencia con el perfil profesional."

Por otro lado, los resultados evidencian que en la mayoría de los casos los objetivos generales no son compatibles con el enfoque metodológico, propuesto en el acápite de las "recomendaciones metodológicas al docente" en cada programa, ya que según este enfoque se deben desarrollar las 4 habilidades simultáneamente.

Este hecho es ratificado por Batista, Salazar \& Febres (2001), quienes expresan: "En el enfoque comunicativo el objeto de enseñar una segunda lengua consiste en desarrollar equilibradamente las cuatro destrezas de la lengua (escuchar-hablar y leerescribir) haciendo énfasis en el carácter funcional más que en aspectos gramaticales y situacionales."

En cuanto a los objetivos específicos, se encontró que están planteados por temas y existe coherencia entre los objetivos específicos de una unidad a otra en el mismo programa. No así de un programa a otro, ya que al ser diseñados en base a textos que no llevan una secuencia lógica, hace que el proceso enseñanza-aprendizaje esté fraccionado. 


\section{Formas de evaluación sugeridas en los programas de inglés}

Otro hallazgo radica en relación a las formas de evaluación que se le sugieren al docente, el cual es muy tradicional, ya que se le concede más peso a los exámenes parciales (60\%) y un $40 \%$ a la evaluación sistemática, no se hace referencia a las evaluaciones diagnósticas y formativas. Lo cual se contradice con lo que plantea el modelo pedagógico que: "la evaluación del aprendizaje es un proceso continuo que debe acompañar el proceso de enseñanza ya que ambos son inseparables." Y agrega que se debe "partir del interés del estudiante en la medida en que su motivación es el motor que impulsa el aprendizaje." (URACCAN, 2004).

\section{Bibliografía en el diseño de los programas de inglés}

En cuanto a la bibliografía está desactualizada. Los libros de textos que se sugieren como textos básicos de los programas son Intercom Book No. 1, 2, 3, 4 editados en 1990 y el Super Goal Book No. 2, y 5, editados en el 2003. Además, el enfoque metodológico del libro de texto Intercom es gramatical, por lo tanto es muy pobre en actividades comunicativas, no consta de CDs para el audio. Por el contrario la serie Super Goal está diseñado bajo un enfoque comunicativo, es ilustrado, colorido, fácil de usar por el estudiante, contiene CDs de audio. Sin embargo, sólo el programa de inglés VI está diseñado en base al libro No. 5 de esta serie, otros programas sólo toman 1 o 2 unidades de estos libros.

Por otra parte, el CNU (2008) expresa que se considera desactualizada la bibliografía propuesta en un programa de asignatura cuando esta tiene más de 10 años de haber sido publicada.

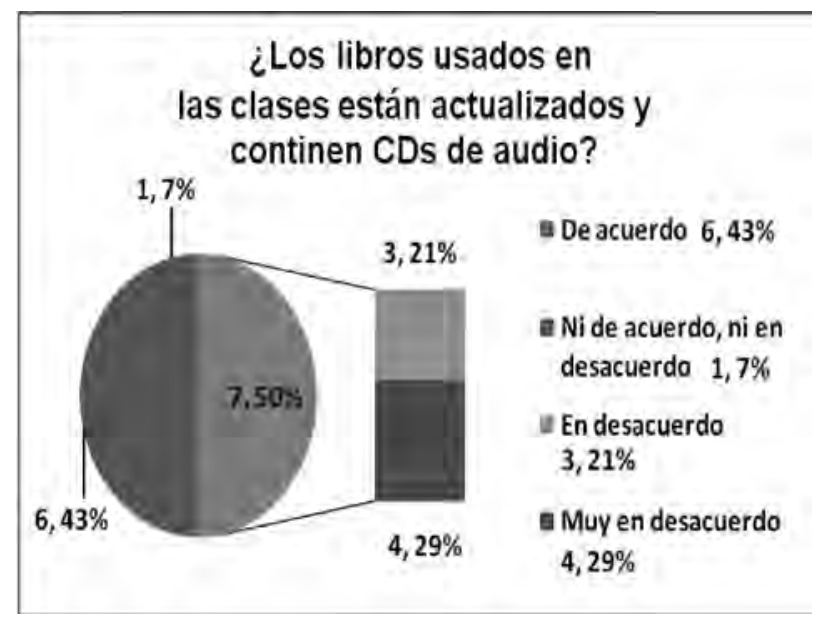

Figura No. 1. Porcentaje de estudiantes en desacuerdo con textos desactualizados 
El 50\% de los estudiantes encuestados, por otro lado, concuerdan con los planteamientos anteriores al considerar que los libros de textos utilizados para las clases de inglés están desactualizados y no existen $C D$ s de audio para facilitar el aprendizaje del idioma inglés. Sin embargo, hay un $43 \%$ que considera que tienen acceso a libros actualizados para las clases de inglés, es posible que se refieran a los textos Super Goal, de los cuales se mencionó que son los únicos libros que están actualizados y que concuerdan con el enfoque metodológico sugerido en los programas.

Además, Magna 2009, afirma que:

los materiales didácticos son fundamentales y cuando se refiere a los libros de textos hace alusión a tres características: procedencia, condiciones de compra, contenido lingüístico, refiriéndose a que los libros de textos deben estar basados en enfoques comunicativos y no en un enfoque tradicional de gramática y traducción, ya que muchas veces esto desmotiva al estudiante.

Se infiere que los textos sobre los cuales se diseñan los programas de una determinada asignatura, en este caso inglés, deben estar actualizados. El enfoque de instrucción debe estar de acuerdo a lo que se quiere lograr en el estudiantado, al disponer de material de audio y que esté a disposición y alcance de los estudiantes.

\section{Tiempo dedicado al estudio del inglés}

Por último, otro aspecto que no concuerda en los programas de inglés es la distribución del tiempo dedicado para el desarrollo de cada unidad. Además, si se suman las 64 horas que contiene cada uno de los 6 programas de inglés de la carrera en Administración de Empresas, se totalizan 384 horas.

No obstante, según los docentes de inglés que han desarrollado estos programas y los docentes que participaron en este estudio, advierten que con este número de horas y con los programas actuales algunos estudiantes logran alcanzar un nivel de comprensión de información escrita, pero no una competencia de comunicación oral fluida. Agregan que algunos estudiantes son capaces de articular ideas para comunicar cierta información, pero no son capaces de interactuar en cualquier situación cotidiana (Entrevista a docentes, 6-12-2009).

A este respecto, una de las autoridades de la URACCAN y especialista en la enseñanza del idioma inglés, considera que:

600 horas debería ser suficiente para que un estudiante de idiomas desarrolle una competencia comunicativa a nivel intermedio, siempre y cuando el enfoque de enseñanza sea comunicativo. Los resultados son proporcionales al número de horas recibidas, la intensidad y frecuencia del curso, al enfoque utilizado, la competencia 
científica y pedagógica del instructor, el grado de interés o motivación del estudiante y sus aptitudes innatas, entre otras. (Entrevista, 10-01-2010).

Otra de las autoridades agrega que es pertinente e indispensable aumentar al menos a 8 programas de inglés en la carrera en Administración de Empresas. Afirma que de esta manera se lograría que los profesionales de esta licenciatura logren un nivel mayor en el idioma inglés y tengan más oportunidades laborales y académicas en el futuro (Entrevista, 26-11-2009).

\section{Condiciones para el aprendizaje del inglés}

La URACCAN Recinto Nueva Guinea cuenta con instalaciones propias. Cuenta con 4 edificios donde se ubican las aulas de clase. Además, existe una biblioteca equipada con la bibliografía básica de cada una de las carreras que se ofertan en este campo universitario, aquí el estudiantado de todas las carreras tienen acceso al servicio de internet gratuito para sus investigaciones. Por otro lado, existe un laboratorio de informática que provisionalmente se ha adecuado para las clases de inglés, aunque se utiliza con este fin sólo en aquellos espacios libres cuando no está ocupado con clases de informática.

La figura No. 2 muestra que un número significativo de los discentes concuerda que el aula de clases sólo ofrece las condiciones básicas (pizarra, pupitres, energía eléctrica, iluminación, silla y mesa para el docente y el espacio mismo del aula). Reconocen que la URACCAN ha hecho muchos esfuerzos para ofrecer las mejores condiciones. No obstante, consideran que las aulas no están ambientadas para facilitar el proceso de aprendizaje del inglés.

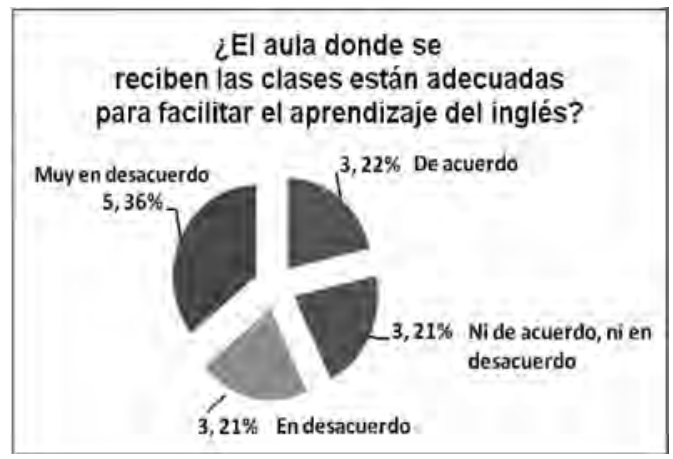

Figura No. 2. Aulas habilitadas para el aprendizaje.

Por otro lado, encontramos que un $22 \%$ considera que las aulas llenan sus expectativas para el aprendizaje del idioma inglés. Aquí se refleja que los estilos de aprendizaje varían de un individuo a otro, es decir, mientras unos aprenden sólo con ciertas condiciones otros necesitan un ambiente más propicio para el aprendizaje. 


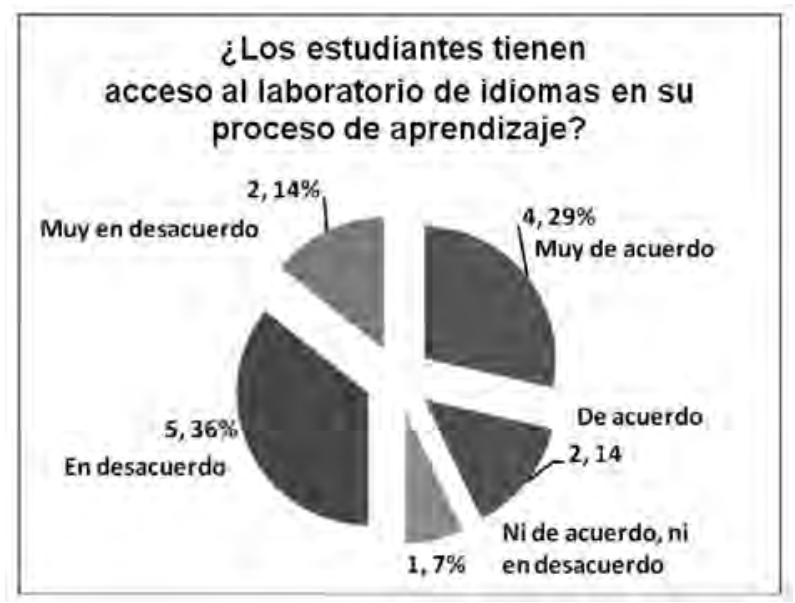

Figura No. 3. Laboratorio para el aprendizaje del inglés.

El 50\% del estudiantado concuerda con Cárdenas (2002), al confirmar que la función del laboratorio de idiomas, bien acondicionado, es indispensable durante el aprendizaje del inglés en la carrera de Administración de Empresas. No obstante, manifiestan no tener acceso a este medio en su aprendizaje. La figura muestra además que un $43 \%$ expresaron que hacen uso del laboratorio de idiomas, esto se explica porque los docentes aprovechan algunos espacios libres para hacer uso del laboratorio de informática para conducir ciertas lecciones, pero esto no es constante.

El personal docente de inglés reconoce el esfuerzo que hace URACCAN Nueva Guinea para brindar los mejores ambientes estudiantiles. No obstante, las aulas de clase sólo brindan las condiciones mínimas y concuerdan con los planteamientos anteriores sobre la importancia de destinar un espacio exclusivo para las clases de inglés. Hacen referencia también al papel que juega el laboratorio de idiomas para el desarrollo de las 4 habilidades del idioma inglés (Entrevista a docentes, 06-12-2009).

A este respecto, en la perspectiva interaccionista para el aprendizaje de una segunda lengua, Williams \& Burden (1999) citados por Martínez (2003), sostienen que la influencia del entorno en el que se desenvuelve el proceso de aprendizaje es fundamental para todo aprendiz.

\section{Medios y materiales didácticos para la enseñanza-aprendizaje del inglés}

La figura No. 4 muestra que el $65 \%$ del estudiantado manifiesta no contar con los materiales básicos para el aprendizaje del idioma inglés, libros de texto con CDs, de audio y diccionarios. Las razones por la que se da este hecho varían en relación a las condiciones de cada estudiante. En algunos casos por razones económicas y en otros por razones de interés personal sobre el aprendizaje del idioma. Los docentes manifiestan 
que si bien es cierto que no se cuenta con bibliografía original, ellos elaboran folletos que contienen los temas orientados por los programas.

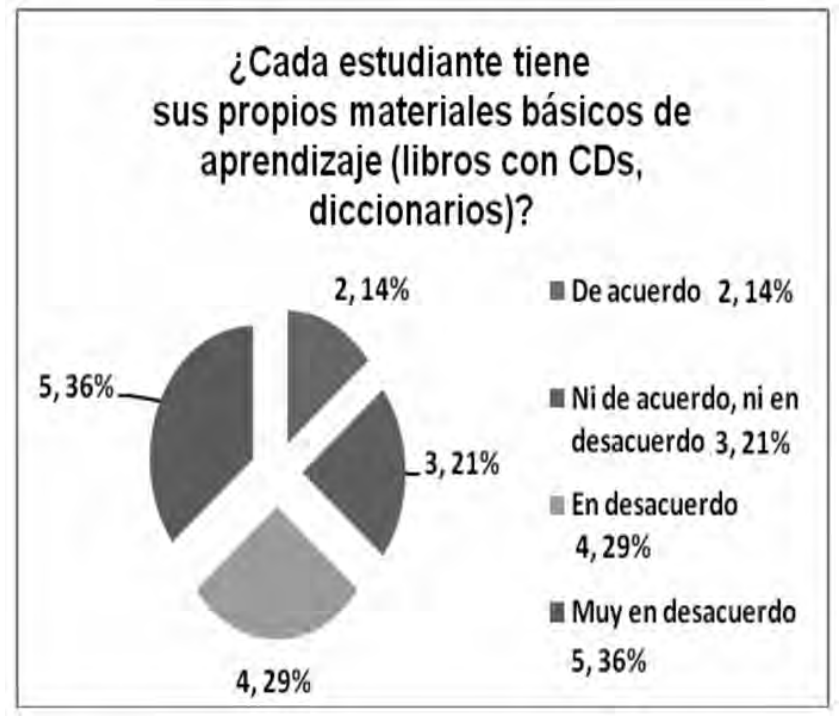

Figura No. 4. Materiales de estudio.

Además, agregan que tienen acceso a otros medios y materiales en el proceso de enseñanza-aprendizaje del idioma inglés. Entre estos destacan libros de gramática del inglés, diccionarios inglés-español, televisor, grabadora, data show, rota folio y servicio de internet (Entrevista a docentes, 6-12-2009).

\section{Motivaciones expresadas en torno al aprendizaje del inglés}

Magna (2009), resalta que el éxito en la adquisición de segundas lenguas: La motivación es condición indispensable para el aprendizaje del idioma meta y afirma que en el área adquisición-aprendizaje la relación motivación-éxito es directa.

A este respecto, este estudio demuestran que el personal docente ha desarrollado los programas de inglés que contempla el plan de estudios de la carrera en Administración de Empresas, afirman que en las aulas de clase tratan que el proceso educativo sea lo más activo y creativo posible (Entrevista a docentes, 6-12-2009).

Se encontró que un porcentaje significativo del estudiantado ratifica lo expresado por los docentes al expresar que los maestros utilizan estrategias variadas para hacer dinámico y activo el proceso de enseñanza-aprendizaje del inglés. 


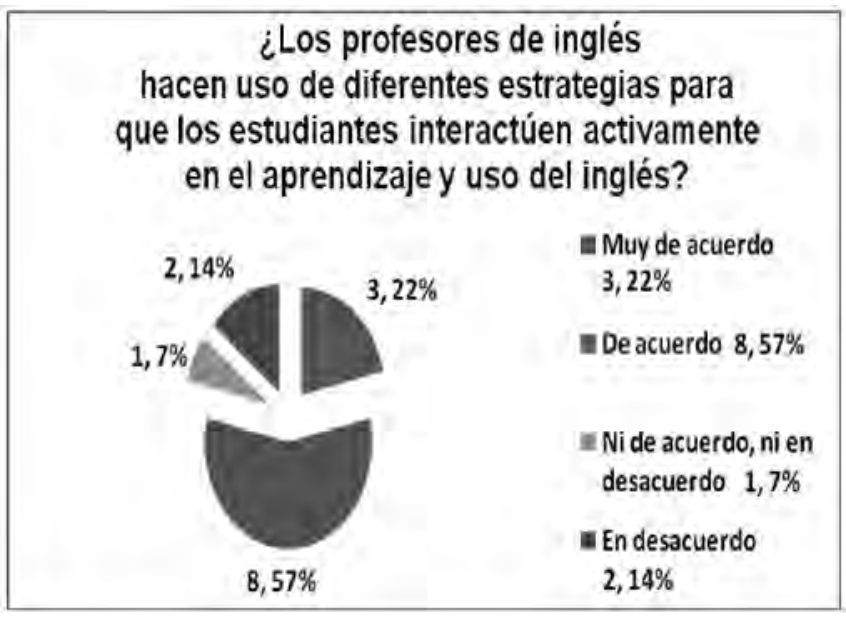

Figura No. 5. Estrategias en el aprendizaje del inglés.

No obstante, una de las autoridades de la URACCAN, especialista en la enseñanza del idioma inglés, resalta que el interés y motivación que muestre el estudiante como tal juega un papel determinante, es decir, que los estudiantes deben de contribuir de forma significativa en su propio aprendizaje y aprovechar cada espacio que se le brinda para practicar y usar la lengua que aprende. Agrega que para la URACCAN es importante que sus estudiantes se gradúen hablando una segunda lengua, ya que de esta manera tendrán más y mejores oportunidades laborales (Entrevista, 10-01-2010).

\section{Conclusiones}

El análisis de los resultados permitió llegar a las siguientes conclusiones: La URACCAN consciente de la importancia de que el estudiantado se gradúe en la carrera en Administración de Empresas y dominen una segunda lengua, en este caso inglés, ha emprendido esfuerzos en el diseño curricular, capacitación docente y creación de condiciones para que el aprendizaje del idioma inglés sea efectivo y significativo. Además, este hecho comprueba que esta institución de Educación Superior busca brindar una educación de calidad y acorde a los cambios que marca el contexto local, nacional e internacional.

A pesar de los esfuerzos que se han realizado para mejorar los 6 programas de inglés que forman parte del plan de estudios de la licenciatura en Administración de Empresas, se pudo comprobar que es necesario emprender un proceso de readecuación de los programas, diseñándolos en base a una bibliografía actualizada y con un enfoque comunicativo. 
Las 384 horas dedicadas al proceso de enseñanza-aprendizaje del idioma inglés y con los programas, no es suficiente para que los estudiantes logren alcanzar una competencia comunicativa, que les permita interactuar en situaciones cotidianas o académicas.

Los expertos concuerdan que el nivel de aprendizaje de una segunda lengua es relativo al número de horas de instrucción y exposición a la misma y a las condiciones que se creen para facilitar el proceso de aprendizaje; pero, sin obviar el rol del docente, medio en que se da el aprendizaje y lo más importante el interés y motivación del aprendiz.

El docente juega un rol determinante en el aula de clase, en la creación de un ambiente de interacción en el cual se involucren los discentes de forma activa, contribuyendo a fijar un aprendizaje significativo del idioma meta.

\section{Lista de referencias}

Batista, J., Salazar, L., \& Febres, M. (2001). Desarrollo de Destrezas Lectoras en L2 desde una perspectiva Constructivista. Revista OMNIA, Año 7, No. 1 y 2:157-192.

CNU (2008). Guía para la Presentación de Proyectos Institucionales de Educación Superior en Nicaragua. Nicaragua. Comisión Académica, Consejo Nacional de Universidades.

Magna, A. (2009). Incidencia de los factores afectivos en la enseñanza/aprendizaje de lenguas extranjeras: Caso del español en Camerún. Ogigia, revista electrónica de estudios hispánicos, 19-28. ISSN: 1887-3731 Universidad de Yaundé I, Camerún.

Martínez, A. (2003). Towards the teaching of foreign languages based on the development of communicative interaction. Facultad de Educación. Universidad de Extremadura. ISSN 1130-0531

Palmero, R. M. L. (2004). La teoría del aprendizaje significativo. Pamplona, Spain. Centro de Educación a Distancia (C.E.A.D.). C/ Pedro Suárez Hdez, Santa Cruz de Tenerife.

URACCAN (2004a). Modelo Pedagógico Institucional.

URACCAN (2003b). Currículum de la Carrera de Administración de Empresas. 